Article

\title{
Non-Random Sister Chromatid Segregation in Human Tissue Stem Cells
}

\author{
Krishnanchali Panchalingam ${ }^{1}$, Laura Jacox ${ }^{2} \oplus$, Benjamin D. Cappiello ${ }^{3}$ and \\ James L. Sherley ${ }^{4, *}$ \\ 1 eGenesis, Cambridge, MA 02139, USA; krisha.panchalingam@egenesisbio.com \\ 2 Divisions of Oral and Craniofacial Science \& Craniofacial and Surgical Care, Adams School of Dentistry, \\ University of North Carolina, Chapel Hill, NC 27599, USA; ljacox@live.unc.edu \\ 3 AxoSim, Inc., New Orleans, LA 70112, USA; ben.cappiello@axosim.com \\ 4 Asymmetrex, LLC, Boston, MA 02130, USA \\ * Correspondence: jsherley@asymmetrex.com; Tel.: +1-617-990-6819
}

Received: 14 October 2020; Accepted: 11 November 2020; Published: 13 November 2020

check for updates

\begin{abstract}
The loss of genetic fidelity in tissue stem cells is considered a significant cause of human aging and carcinogenesis. Many cellular mechanisms are well accepted for limiting mutations caused by replication errors and DNA damage. However, one mechanism, non-random sister chromatid segregation, remains controversial. This atypical pattern of chromosome segregation is restricted to asymmetrically self-renewing cells. Though first confirmed in murine cells, non-random segregation was originally proposed by Cairns as an important genetic fidelity mechanism in human tissues. We investigated human hepatic stem cells expanded by suppression of asymmetric cell kinetics (SACK) for evidence of non-random sister chromatid segregation. Cell kinetics and time-lapse microscopy analyses established that an ex vivo expanded human hepatic stem cell strain possessed SACK agent-suppressible asymmetric cell kinetics. Complementary DNA strand-labeling experiments revealed that cells in hepatic stem cell cultures segregated sister chromatids non-randomly. The number of cells cosegregating sister chromatids with the oldest "immortal DNA strands" was greater under conditions that increased asymmetric self-renewal kinetics. Detection of this mechanism in a human tissue stem cell strain increases support for Cairns' proposal that non-random sister chromatid segregation operates in human tissue stem cells to limit carcinogenesis.
\end{abstract}

Keywords: hepatic stem cell; SACK; xanthosine; xanthine; hypoxanthine; asymmetric cell kinetics; chromosome segregation; sister chromatids; non-random segregation; immortal DNA

\section{Introduction}

Recent studies confirm that mammalian chromosomes can segregate non-randomly when cells divide with asymmetric self-renewal kinetics [1-10]. Non-random chromosome segregation violates the classical rules of random segregation and independent assortment of replicated mammalian sister chromatids during mitosis. This alternative mode of genomic DNA segregation was first suggested by Cairns [11] as a unique property of mammalian tissue stem cells when they underwent asymmetric self-renewal divisions for tissue cell homeostasis. Cairns envisioned that asymmetrically self-renewing tissue stem cells cosegregated to themselves all, and only, the sister chromatids with the older template DNA strands from semiconservative DNA replication. The sister chromatids with the younger template DNA strands assorted to the non-stem cell daughter cell. In this way, Cairns proposed that tissue stem cells could preserve their genetic fidelity by avoiding the accumulation of mutations caused by DNA replication errors [11-13]. Cairns named the cosegregated older template DNA strands "immortal DNA strands" to signify their predicted extended lifetime in tissue stem cells [11]. 
Previously, we performed mathematical modeling studies that indicated that non-random sister chromatid segregation as proposed by Cairns would have a large effect—on the order of 1000-fold-in reducing the accumulation of spontaneous DNA mutations in tissue stem cells [14]. Our earlier studies also showed that the TP53 tumor suppressor protein is a major regulator of both tissue stem cell asymmetric self-renewal and non-random segregation, which are coupled by a yet to be discovered cellular mechanism [1-5,15]. Given the TP53 gene's identity as the most commonly mutated gene in many diverse human cancers, we have suggested that an important overlooked carcinogenic effect of TP53 gene mutation is the loss of tissue stem cell genetic fidelity and asymmetric self-renewal control [14-16].

Although its postulated reduction of mutations in tissue stem cells has not been investigated, non-random sister chromatid segregation has been described in several cellular systems. These include engineered cell lines that undergo asymmetric self-renewal [1-5,9], natural tissue stem cells in cell culture [5-10,17,18], tissue stem cells in animal tissues in vivo [19-24], and at low levels in cancer stem cell lines [25-29]. Evidence for non-random segregation involving either all or only a subset of chromosomes has been reported for Aspergillus nidulans [30] and Drosophila melanogaster [31,32], respectively. There are also several reports of low or undetectable levels of non-random segregation in mammalian and insect tissue stem cell compartments [33-38]. Cairns based his originating theoretical ideas on non-random immortal DNA strand inheritance on the seminal experimental studies of Lark and coworkers $[39,40]$, who identified non-random sister chromatid segregation in cultured primary murine embryonic tissue cells [41] and plant root tip cells [42].

With two exceptions $[9,10]$, all previous demonstrations of non-random sister chromatid segregation by mammalian tissue cells were performed with murine cells or cancerous human cells. In a first brief account, we showed evidence of non-random segregation in normal human muscle cell cultures enriched for Pax7-expressing cells [9]. Pax7 is molecular expression marker for human muscle satellite stem cells. In the same report, we established the asymmetric nuclear localization of the histone variant H2A.Z as a prophase molecular marker of cells undergoing non-random sister chromatid segregation (i.e., "H2A.Z asymmetry"). The Pax7 cell-enriched cultures were shown to contain rare cells that exhibited H2A.Z asymmetry. Importantly, the frequency of cells exhibiting H2A.Z asymmetry was decreased by supplementation of cultures with xanthosine, a guanine ribonucleotide precursor. Xanthosine shifts tissue stem cell kinetics from asymmetric self-renewal to symmetric self-renewal [6-8,43-46].

In a second later report [10], H2A.Z asymmetry was detected in cultures of a human hepatic stem cell strain, 12(3), derived by the method of suppression of asymmetric cell kinetics (SACK) [10,43,44,47]. The SACK method has been described for its use to expand and propagate rat [43], murine [6], and human $[10,46,47]$ tissue stem cell strains, including from liver cell lineages $[10,43,47]$, by using supplementation with specific purine bases and nucleosides to shift tissue stem cells for asymmetric self-renewal to symmetric self-renewal. Xanthosine, mentioned above, is an example of a SACK agent. It was used for the expansion and propagation of the 12(3) cell strain [10,47]. The reported detection of H2A.Z asymmetry in 12(3) cell cultures was indicative of the presence of cells undergoing non-random sister chromatid segregation [10].

In the present report, we provide the full experimental basis for the earlier, brief report of evidence for non-random sister chromatid segregation in SACK agent-expanded human hepatic tissue stem cells. The non-randomly segregating cells are defined as hepatic stem cells based on the xanthosine-dependency of their asymmetric self-renewal and associated non-random sister chromatid segregation. These findings are an important contribution to advancing Cairns' essential hypothesis that the rate of human carcinogenesis is limited by an immortal DNA strand cosegregation mechanism that functions to limit mutation accumulation in asymmetrically self-renewing human tissue stem cells. 


\section{Materials and Methods}

\subsection{SACK Expansion and Propagation of Human Hepatic Stem Cell Strains}

The method for expansion and propagation of human hepatic stem cell strains by the SACK method has been described and patented $[10,47]$. Strain derivations were initiated with the low-speed $50 \times g$ non-parenchymal cell supernatant fraction prepared from human liver specimens from either parental-consented, deceased traumatically injured donors or consented surgical discards. These specimens were supplied by Cambrex (Rutherford, NJ, USA) and used to derive the described cell strains when the authors were investigators at the Massachusetts Institute of Technology (Cambridge, MA, USA). The reported studies were approved by the MIT Committee on the Use of Humans as Experimental Subjects under COUHES\# 040300449.

Briefly, cells in primary human liver non-parenchymal cell fractions were seeded at 5 million viable cells per $10-\mathrm{cm}^{2}$ dish in Dulbecco's modified Eagle's medium (DMEM) supplemented with 1\% dialyzed fetal bovine serum (DFBS; JRH Biosciences, Lenexa, KS, USA), $20 \mathrm{ng} / \mathrm{mL}$ epidermal growth factor (EGF; Invitrogen, Carlsbad, CA, USA), $0.5 \mathrm{ng} / \mathrm{mL}$ Transforming Growth Factor- $\beta$ (TGF- $\beta$; Sigma Chemical Co., St. Louis, MO, USA), and $1.5 \mathrm{mM}$ of the respective SACK agent (hypoxanthine (Hx), xanthine $(\mathrm{Xn})$, or xanthosine (Xs), purchased from Sigma Chemical Co.) as described in Results. Adherent cells were maintained in a $37{ }^{\circ} \mathrm{C}$ incubator with a $5 \% \mathrm{CO}_{2}$ atmosphere for 3-7 weeks, replacing cultures with fresh medium weekly. After this period, cells were gently rinsed with Hank's balanced salt solution (HBSS), trypsinized to remove, and all recovered cells were transferred to a single well of a 24-well plate with DMEM supplemented with 10\% DFBS and $1.5 \mathrm{mM}$ of the respective SACK agent used to initiate the culture. The 24-well culture maintains a high cell density, which is also an important factor for SACK [48]. Culture was continued in the 24-well format with a medium change after 1-2 days to remove dead cells and cellular debris. When 24-well cultures reached confluence, they were further expanded in the respective medium in the same manner with progressive transfers into one well of a 6-well plate and then into a $75-\mathrm{cm}^{2}$ flask.

When the first $75-\mathrm{cm}^{2}$ culture reached confluence, it was designated as the Passage 0 (P0) founder culture. Four fifths of the cells from the P0 culture were aliquoted into $4 \times$ cryo-vials and cryopreserved at $-130{ }^{\circ} \mathrm{C}$ using the respective culture medium supplemented with $10 \%$ DMSO. The remaining one fifth of cells were used to continue propagation of cell strains. During propagation studies, cultures were passaged within a day of attaining confluence; and at least $1 / 5$ of the harvested cells were always passaged to maintain cells at an optimal high density for SACK. Cryopreserved stock vials were made at semiregular intervals throughout the propagation of new cell strains.

To derive clonal strains, 100 cells were plated per $10-\mathrm{cm}^{2}$ dish in Xs-supplemented medium $(1.5 \mathrm{mM})$ and cultured for two weeks to allow formation of cell colonies. Individual cell colonies were ring-cloned, transferred to one well of a 24-well plate, and expanded and propagated as initial parental cell strains were, except in culture medium not supplemented with EGF and TGF- $\beta$. Stable clones of YFP-expressing fluorescent 12(3) cells were derived as previously described [49].

\subsection{Colony Formation Studies}

Colony formation studies to evaluate the SACK agent dependency of cell strains were performed as previously described [10].

\subsection{Colcemid Arrest Assay}

For the colcemid arrest assay, $5 \times 10^{4}$ cells were plated in $150-\mathrm{cm}^{2}$ flasks in the required medium. After $48 \mathrm{~h}$ of culture, colcemid (Sigma Chemical Co.) was added to a final concentration of $100 \mathrm{ng} / \mathrm{mL}$. Culture was continued for $24 \mathrm{~h}$, and cells were harvested by collecting floating cells and removing adherent cells by trypsinization. After fixation in 70\% ethanol, the harvested cells were stained with $50 \mu \mathrm{g} / \mathrm{mL}$ propidium iodide and treated for $30 \mathrm{~min}$ at $37^{\circ} \mathrm{C}$ with $1 \mathrm{mg} / \mathrm{mL}$ RNase (Sigma Chemical Co.). Flow cytometry analysis was performed as previously described [10]. 


\subsection{Time-Lapse Microscopy Analyses}

A Zeiss Observer.Z1 microscope-equipped with an Incubator XL S1 system, with additional $\mathrm{CO}_{2}$ Module S1, TempModule S1, and Heating Unit XL S1—was used to perform time-lapse analyses. AxioVision 4.7.2. software was used to analyze collected image data. On stage incubator conditions were $37{ }^{\circ} \mathrm{C}, 5 \% \mathrm{CO}_{2}$ atmosphere, and optimized humidity. The image-capture was configured to take one picture in multiple locations of each well every $5 \mathrm{~min}$. Time-lapse images were acquired continuously over $4-5$ day periods, creating videos of 1152 and 1442 images. Images were captured in the phase-contrast mode.

Using the AxioVision software with Adobe Illustrator software, each of the cells on the first frame of videos was labeled in order to track them throughout the four to five day period. The cells were manually tracked by observing changes in their location from frame to frame. Three events were scored from one frame to the next: cell movement, the cell division, or cell apoptosis. Each identified cell was tracked from the first frame until the final frame or until apoptosis. When a division took place, the daughter cells were tracked until their final frame or another division. This analysis process was repeated for a large sample of cells within the field of view.

To estimate cell generation times, the precise time was measured between the observed production of a cell by a mitosis event and its own subsequent mitosis. A graphical representation of cell pedigrees was designed using the Adobe Creative Suite.

\subsection{Sister Chromatid Segregation Analyses}

Label exclusion assays were performed with Hoechst dye 33258 and quantified by fluorescence microscopy as previously described [5,6]. To perform label retention analyses, strain 12(3) cells were plated at a density of 1000 cells per $1-\mathrm{cm}^{2}$ in culture slides (BD Pharmingen Biosciences, San Diego, CA, USA) in Xs-supplemented medium (1.5 mM). After $24 \mathrm{~h}$ of culture, the culture medium was replaced with fresh Xs-supplemented medium containing $20 \mu \mathrm{M}$ bromodeoxyuridine (BrdU). After $24 \mathrm{~h}$ of culture to allow BrdU incorporation under conditions that suppress asymmetric cell kinetics, the medium was removed and slide cultures were rinsed twice with fresh medium lacking BrdU. Xs-free medium (without BrdU) was added to slide cultures to promote asymmetric self-renewal kinetics; and Xs-supplemented medium (without BrdU) was added to slide cultures to maintain conditions that suppress asymmetric self-renewal kinetics. The slide cultures were then cultured for $96 \mathrm{~h}$ (approximately 4 cell generations). After this chase period, cultures were arrested by culture for $24 \mathrm{~h}$ in the respective medium supplemented with $2 \mu \mathrm{M}$ cytochalasin D.

Following cytochalasin D treatment, attached cells were washed with cold, filtered phosphate buffered saline, pH 7.4 (PBS), fixed by treatment for $30 \mathrm{~min}$ with $70 \%$ ethanol chilled to $-20^{\circ} \mathrm{C}$, and then air-dried in the dark.

For immunofluorescence analysis, fixed slides were rinsed with PBS at room temperature for $1 \mathrm{~min}$ and then submerged for $10 \mathrm{~min} 2 \mathrm{M} \mathrm{HCl}$ to denature cellular DNA. After a brief PBS wash, a blocking solution of $0.5 \%$ special form bovine serum albumin (BSA; Sigma Chemical Co., St. Louis, MO, USA) and $0.05 \%$ Tween-20 in PBS was applied for $10 \mathrm{~min}$ in a humidified chamber. Slides were incubated for $2-4 \mathrm{~h}$ with a primary antiBrdU mouse monoclonal IgG antibody solution (MAB 3424; Chemicon International, Temecula, CA, USA) at room temperature. The antiBrdU antibody was diluted 1:100 in the blocking solution and then centrifuged for $4 \mathrm{~min}$ at $16,000 \times g$ to remove particulates before application.

Following primary antibody incubation, slides were washed with blocking solution and then incubated with a secondary polyclonal, FITC-conjugated rabbit antimouse IgG antibody (F0232, Dako, Carpinteria, CA, USA) for $45 \mathrm{~min}$. The secondary antibody was diluted 1:200 in blocking solution to a final concentration of $2.7 \mu \mathrm{g} / \mathrm{mL}$. Slides were then washed three times with blocking solution, three times with PBS, and treated with Vectashield mounting media with DAPI (BD Pharmingen Biosciences). A cover slip was applied to seal slides with a clear nail polish. 
A Zeiss UV microscope was used to image slides. The Open Lab program (Improvision, Lexington, MA, USA) and a Zeiss AxioCam CCD microscope camera were used to photograph the cells using FITC and UV filter sets. Antibody specificity tests were conducted by analyses excluding the primary antiBrdU antibody to insure that detection of nuclear FITC-fluorescence required the primary antibody.

\subsection{Statistical Analyses}

Fisher's exact test (two-tailed) was applied to evaluate the statistical significance of observed differences in sister chromatid segregation patterns related to conditions for SACK. Student's two-tailed $t$-test was applied to evaluate the statistical confidence of differences in cell generation time determined by time-lapse microscopy.

\section{Results}

\subsection{Suppression of Asymmetric Cell Kinetics (SACK) Derivation of Human Hepatic Cell Strain 12(3)}

Strain 12(3) is one of several human hepatic cell strains derived by the SACK method. Table 1 provides a summary of four independent trials of human hepatic cell strain propagation. Each trial compared culture propagation without supplementation with a SACK agent to supplementation at $1.5 \mathrm{mM}$ concentration of one of three agents. These were the purine base xanthine $(\mathrm{Xn})$, or the purine nucleosides hypoxanthine $(\mathrm{Hx})$ or xanthosine $(\mathrm{Xs})$. The primary metabolic effect of these agents is expansion of cellular guanine ribonucleotide pools [44].

Table 1. Comparisons of the extent of cumulative population doublings (CPD) of parallel control and SACK agent-supplemented primary human hepatic cell cultures before terminal division arrest (age and sex of donor indicated and -1 and -2 denote duplicate cultures.).

\begin{tabular}{cccll}
\hline Trial No. & Age (Years) & Gender & Purine & CPD \\
\hline 1 & 6 & M & Control-1 & 24 \\
\hline & & Control-2 & 10 \\
& & Xn & 38 \\
& & Hx & 29 \\
& & Xs & 36 \\
\hline 2 & 14 & F & Control & 10 \\
\hline 3 & & & Hx & 26 \\
\hline & & M & Control & 22 \\
\hline 4 & & Xn & 10 \\
& & & Hx-1 & 30 \\
& & & Hx-2 & 14 \\
& & & Xs-1 & 77 \\
& & & Xs-2 & 21 \\
\hline & & & Control & 16 \\
\hline & & & Xs-1 & 26 \\
& & & Xs-2 & 13 \\
\hline
\end{tabular}

Control non-supplemented cultures underwent a terminal division arrest, which always occurs for primary cultures of normal human tissue cells, after 10-24 cumulative population doublings (CPDs). The extent of propagation obtained with SACK agent-supplemented cultures ranged from 10 to 77 CPDs at the time of the accounting in Table 1, and several strains continued to actively proliferate. From this limited set of culture propagation data, it was not possible to detect significant strain propagation effects related to donor age or sex, or the SACK agent supplemented. Based on its greater extent of proliferation, strain Xs-1 from Trial 3 was selected for subsequent sister chromatid segregation analyses and renamed strain 12(3). 
Figure 1 compares the CPD course of the SACK-agent supplemented expansion of strain 12(3) to the non-supplemented control culture of cells derived from the same donor. On examination of propagating cultures by phase microscopy, SACK agent-supplemented cultures showed denser and healthier cell populations than non-supplemented cultures (compare Figure $2 \mathrm{~b}$ to Figure 2a). Clonal strains initiated with isolated cell colonies of strain 12(3) cells were readily propagated when maintained in medium supplemented with $1.5 \mathrm{mM}$ Xs. Nine independent clonal strains were derived from 12(3) cells that had been cryopreserved at the sixth passage of propagation. As shown in Figure 1, the clonal derivatives recapitulated the CPD history of the parental 12(3) strain. It was not possible to derive clonal derivatives from non-supplemented cell strains.

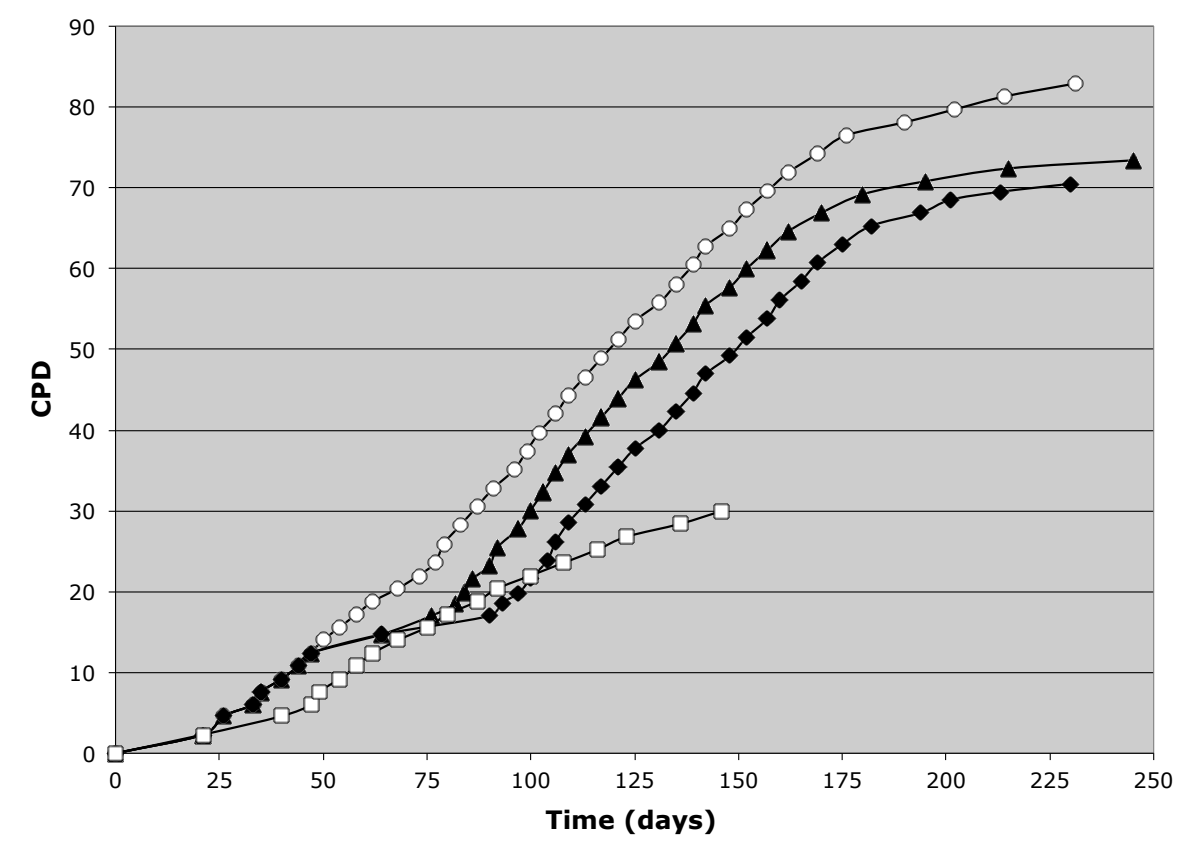

Figure 1. Comparison of the cumulative population doubling (CPD) courses of parallel control and Xs-supplemented primary human hepatic cell cultures. The cultures were serially passaged at the indicated time intervals. Open squares, control with no SACK agent supplementation. Open circles, supplementation with $1.5 \mathrm{mM}$ Xs. Also shown are the subsequent CPD courses of two independent clonally derived strains of the Xs-supplemented parental strain 12(3). Closed triangles, clonal strain 12(3)-C1. Closed diamonds, clonal strain 12(3)-C2. The CPD plots for the clonal strains were extrapolated to the origin culture from the point of their derivation at passage 6 of their parental strain.

To our knowledge, derivation of primary clonal human cell strains from isolated cell colonies has not been accomplished previously. Earlier reports of clonal derivation of human tissue cells are limited to either tumor-derived cells or cells experimentally induced to undergo oncogenic transformation. The ability to produce clonal SACK agent-expanded cell strains indicated that 12(3) cells might also be propagated clonally after genetic engineering. We confirmed this capability by producing stable yellow fluorescent protein (YFP)-expressing, fluorescent 12(3) subclones that could be propagated and cryopreserved like parental 12(3) cells (Figure 2c,d). 

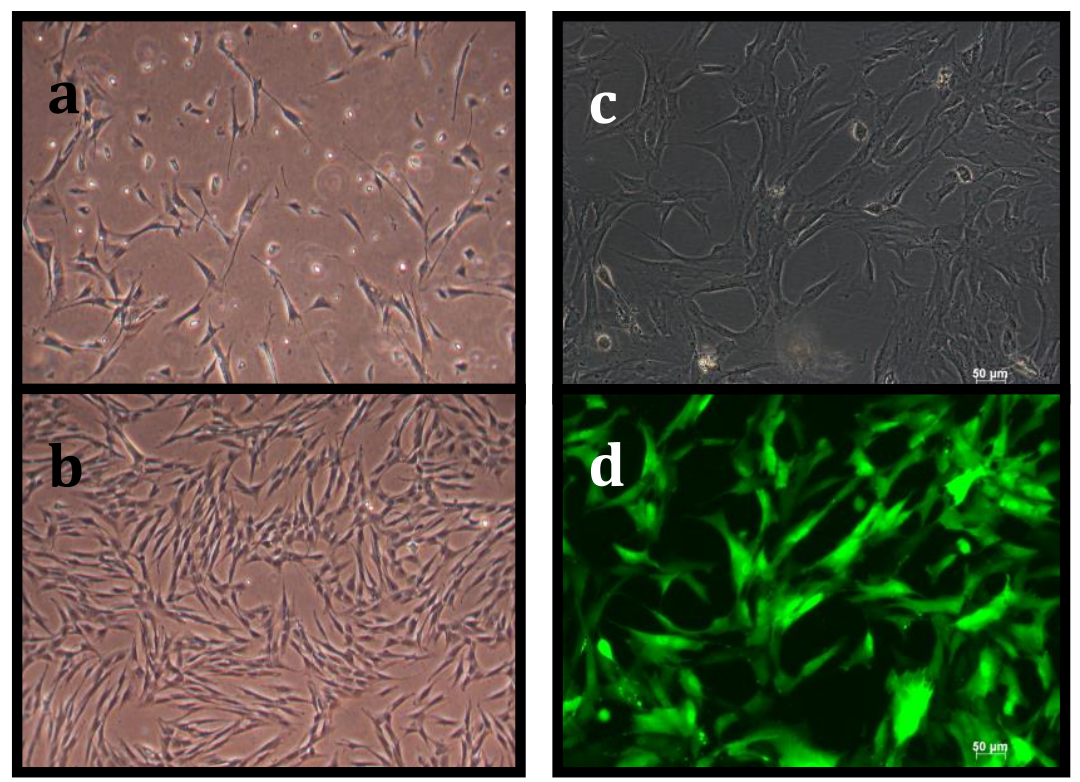

Figure 2. Phase and fluorescence micrographs of cultured human hepatic cells during and after SACK agent-supplemented strain propagation. (a) Control non-supplemented propagation culture (Trial 3 in Table 1). (b) Xs-supplemented (1.5 mM) expansion culture (Trial 3 derivation Xs-1; strain 12(3)). (c) and (d) Stably transfected, YFP-expressing, clonally derivative of SACK agent-expanded strain 12(3) examined by phase and fluorescence microscopy, respectively.

\subsection{Tissue Stem Cell Kinetics Properties of Human Hepatic Cell Strain 12(3)}

SACK agent expansion and propagation of tissue stem cells in culture is due to the ability of guanine ribonucleotide precursors to shift tissue stem cells from asymmetric self-renewal kinetics to symmetric self-renewal kinetics. SACK agent-dependent asymmetric cell kinetics is an important criterion for identification of postnatal and perinatal tissue stem cells, even if they have not been cultured previously in SACK agents [50,51]. Our earlier reports also establish asymmetric self-renewal division as an important requirement for non-random sister chromatid segregation as originally postulated by Cairns [1-10].

Several different analyses were applied to characterize the cell kinetics properties of strain 12(3) cells and their clonal derivatives. Figure 3 data are examples of results from colony formation assays. The majority of cells strains established in SACK agent-supplemented media show a significant SACK agent-dependent increase in colony formation efficiency. This effect was not due to increased plating efficiency, because control medium was replaced with SACK agent-supplemented medium only after cells had $24 \mathrm{~h}$ for initial adherence. Mirroring the greater proliferative capacity of strain 12(3) cultures, isolated individual cells in 12(3) cultures displayed the greatest degree of proliferative response to SACK agent supplementation.

Depending on the tissue type in vivo, the number of cells that constitute the differentiating cell lineages produced by the asymmetric self-renewal divisions of tissue stem cells can vary from a single differentiated cell to many thousands. In vitro, the normal tissue phenotypic architecture of differentiated cell lineages is disrupted, but terminally arrested non-dividing cells are usually produced $[48,51]$. The continuous production of viable, terminally arrested cells in culture is an indicator of the presence of asymmetrically self-renewing tissue stem cells [48,51]. 


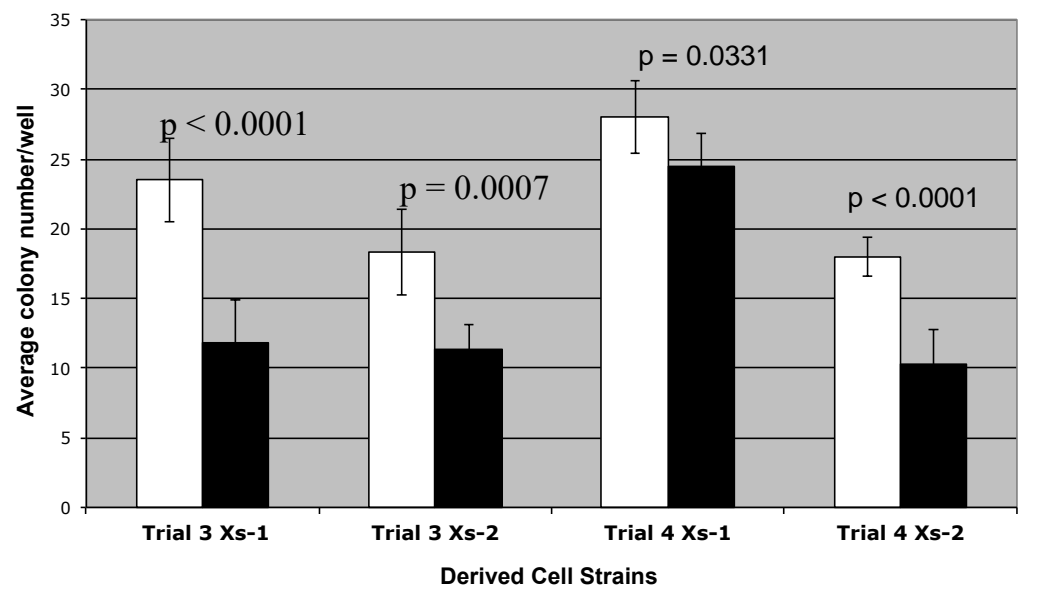

Figure 3. Colony formation analysis to investigate the SACK agent-dependence of primary human hepatic cell strains derived with Xs-supplementation. The average number of cell colonies detected by crystal violet staining after plating cell strains at low colony-forming cell densities ( 300 cells per $25 \mathrm{~cm}^{2}$ ) and then cultured in control (black bars) or Xs-supplemented culture medium (1.5 mM Xs; white bars) for two weeks. The evaluated expansion strains were derived in the respective trial studies listed in Table 1. Error bars = replicate sample SD $(n=6)$.

To investigate the production of non-dividing cells in cultures of SACK agent-propagated cell strains, we developed a flow cytometry colcemid-arrest assay. Colcemid is a microtubule antagonist that causes actively cycling cells to arrest in mitosis of the cell cycle. Flow cytometry can be used to quantify arrested mitotic cells by their distinct 4n DNA content (M phase), compared to G1 phase cells with 2n DNA content, and S phase cells with DNA content greater than $2 \mathrm{n}$ and less than $4 \mathrm{n}$. In cultures of uniformly cycling cells, treatment with colcemid for one cell generation period will result in essentially all cells in the culture arrested in M phase with 4n DNA content. However, if arrested non-cycling cells are being produced in the culture, they will not progress to the M phase arrest. They can be quantified as cells that retain G1 and S DNA content even after a full generation period of culture in colcemid. As shown in Figure 4, when cultured under control Xs-free conditions, strain 12(3) (Figure 4c) and two clonal derivatives (Figure 4d,e) show the production of a high fraction of viable non-cycling cells, primarily arrested in the G1 phase of the cell cycle. Arrest in the G1 phase is commonly observed for differentiated terminally arrested cells. Consistent with their SACK agent derivation basis and the SACK agent dependency of the cell strains' proliferation, Xs supplementation markedly reduced the production of non-cycling terminally arrested cells detected with the colcemid treatment (Figure 4f).

As noted earlier, the continuous production of non-cycling terminally arrested cells in cultures of presenescent human cells is a hallmark of underlying asymmetric self-renewal division by tissue stem cells [48,51]. To identify asymmetrically self-renewing hepatic stem cells in cultures of strain 12(3) cells and to investigate the cell kinetics architecture of their asymmetric non-dividing cell lineages in culture, we performed time-lapse phase microscopy analyses. Figure 5 provides examples of division pedigrees defined as asymmetric self-renewal division lineages. These pedigrees were characterized by a cell that divided to produce single cells that did not divide for the remainder of the $124 \mathrm{~h}$ imaging period. 


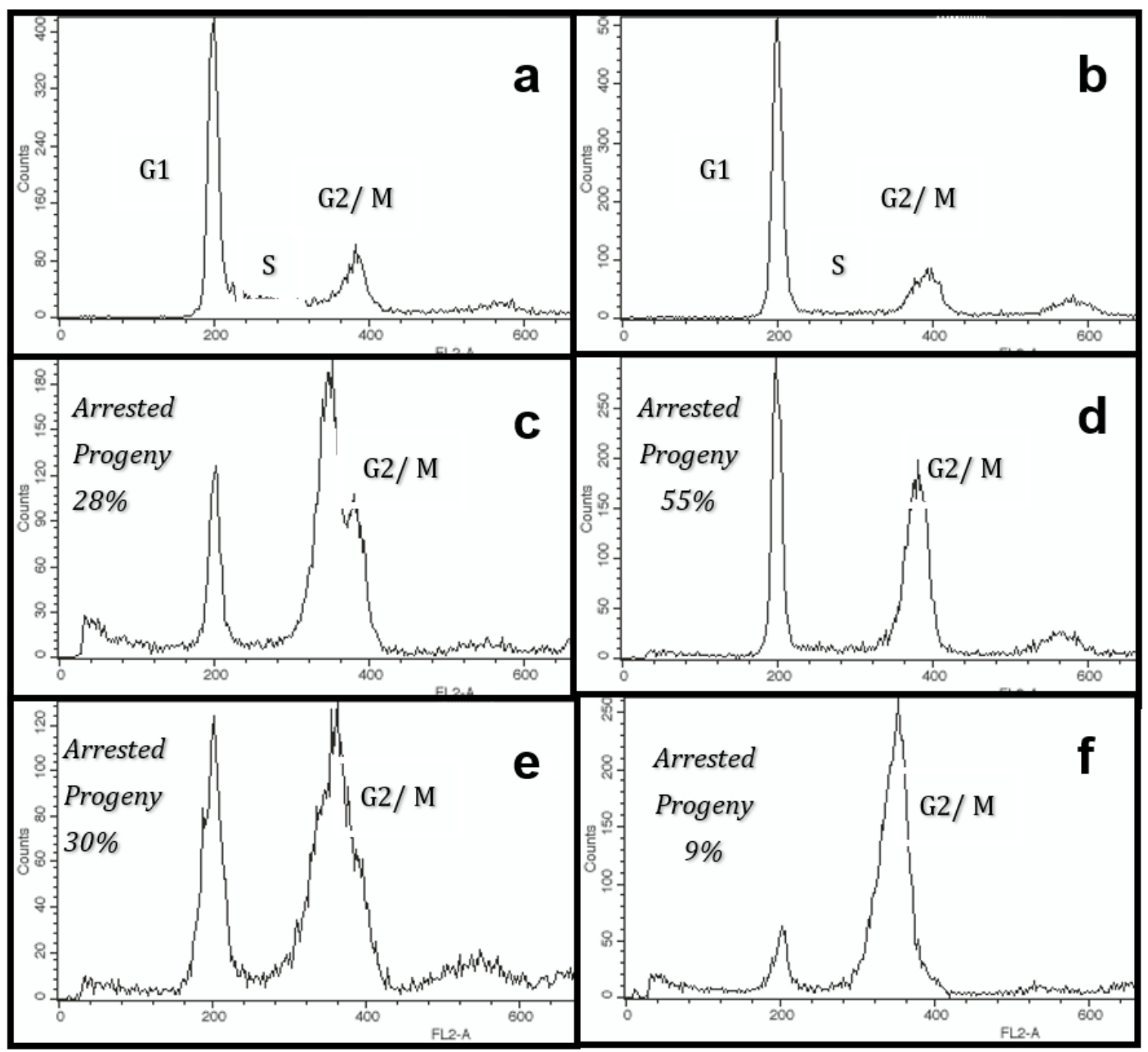

Figure 4. Flow cytometry colcemid arrest assay to detect non-cycling, terminally arrested cells produced in cultures of expanded primary human liver cells. Actively proliferating cultures were maintained for $24 \mathrm{~h}$ in either Xs-free medium $(\mathbf{a}, \mathbf{b})$, Xs-free medium supplemented with colcemid (c-e), or medium supplemented with both Xs $(1.5 \mathrm{mM})$ and colcemid (f). Thereafter, the cells were harvested, fixed, and stained with propidium iodide (PI) for flow cytometry analysis. DNA-PI fluorescence histograms obtained by flow cytometry are shown. The following expanded cell strains were evaluated. (a,c) Parental cell strain 12(3). (b,d) Clonal derivative strain 12(3)-C1. (e,f) Clonal derivative strain 12(3)-C2. Colcemid was used to identify cycling cells based on their progression to a mitotic arrest with G2/M 4n DNA content. Non-cycling cells in the same cultures were identified as cells that persisted with G1 and S phase DNA content without progression to a G2/M amount of DNA content in the presence of colcemid.

Time-lapse imaging was begun for strain 12(3) cells immediately after culture replacement with either Xs-free medium or Xs-supplemented medium $(1.5 \mathrm{mM})$. A total of 65 pedigrees were developed, 35 with Xs supplementation and 30 without. In addition to the described asymmetric self-renewal pedigrees, four other pedigree types were observed: (1) single, terminal-arrested cells that did not divide during the entire imaging period; (2) terminal division cells that divided early, within one generation period on average and produced two daughter cells that did not divide during the remainder of the imaging period; (3) cells that yielded three successive generations of uniformly dividing cells, which were defined as symmetric self-renewal pedigrees; and (4) mixed pedigrees in which one new sister continues a symmetric division lineage while the other adopts an asymmetric lineage in which an arrested cell is produced with each subsequent division. 


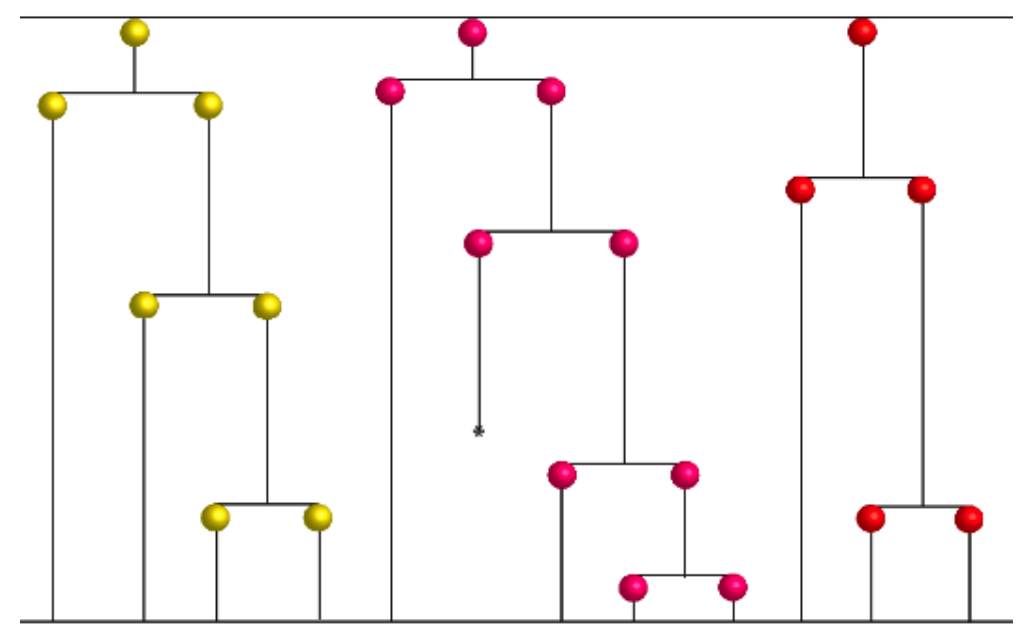

Figure 5. Examples of asymmetric self-renewal division pedigrees determined by time-lapse imaging of SACK agent-derived cell strain 12(3) cultured under Xs-free conditions. The total imaging time is $124 \mathrm{~h}$. Asterisk, a cell that migrated out of the imaging field of view.

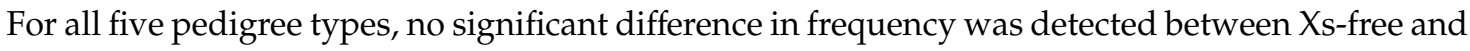
Xs-supplemented medium conditions (Table 2). Similarly, no difference was detected in the frequency of individual asymmetric cell divisions, whether they occurred in asymmetric pedigrees or mixed pedigrees. Symmetric divisions were defined as cell divisions for which both sister cells produced were observed to also divide; whereas asymmetric divisions were defined as divisions for which one sister cell was not observed to divide after one generation period or longer following the division of its sister cell. In the Xs-free medium, $30 \%$ of divisions were asymmetric $(n=40)$; and in the Xs-supplemented medium, $32 \%$ were asymmetric $(n=38)$. There was no significant difference in the mean generation of times of informative cell divisions during the imaging period (Xs-free, $29 \pm 11 \mathrm{~h}$; Xs-supplemented, $31 \pm 15 \mathrm{~h})$.

Table 2. Frequency of different cell division pedigree types observed for strain 12(3) cells by time-lapse microscopy.

\begin{tabular}{lcc}
\hline & \multicolumn{2}{c}{ Time-Lapse Culture Condition } \\
\cline { 2 - 3 } Pedigree Type & Xs-Free & Xs-Supplemented \\
\hline Asymmetric & $7 \%$ & $9 \%$ \\
Terminal Cell & $23 \%$ & $37 \%$ \\
Terminal & $13 \%$ & $11 \%$ \\
Division & $33 \%$ & $17 \%$ \\
Symmetric & $23 \%$ & $26 \%$ \\
Mixed & & \\
\hline
\end{tabular}

\subsection{Detection of Non-Random Sister Chromatid Cosegregation by Human Hepatic Strain 12(3) Cells under} Conditions that Promote Asymmetric Self-Renewal Kinetics

Beyond the derivation of the cells from human liver tissue specimens, previous reports have confirmed the phenotypic identity of strain 12(3) cells as hepatic precursor cells, which includes production and secretion of human albumin and alpha-fetoprotein $[10,47]$. The studies presented here, thus far, provide several independent types of cell kinetics evidence of their tissue stem cell identity. The current state of knowledge dictates that non-random sister chromatid segregation is an exclusive property of tissue stem cells. As such, detection of cells undergoing Xs-dependent non-random sister chromatid segregation in strain 12(3) cultures would, by itself, independently support verification of the cells as a hepatic tissue stem cell strain. 
Two different well-established experimental strategies were employed to investigate the possibility of non-random sister chromatid segregation by asymmetrically dividing hepatic stem cells in cultures of SACK agent-derived 12(3) cells. The two strategies, label exclusion and label retention, are diagrammed and described in Figure 6.

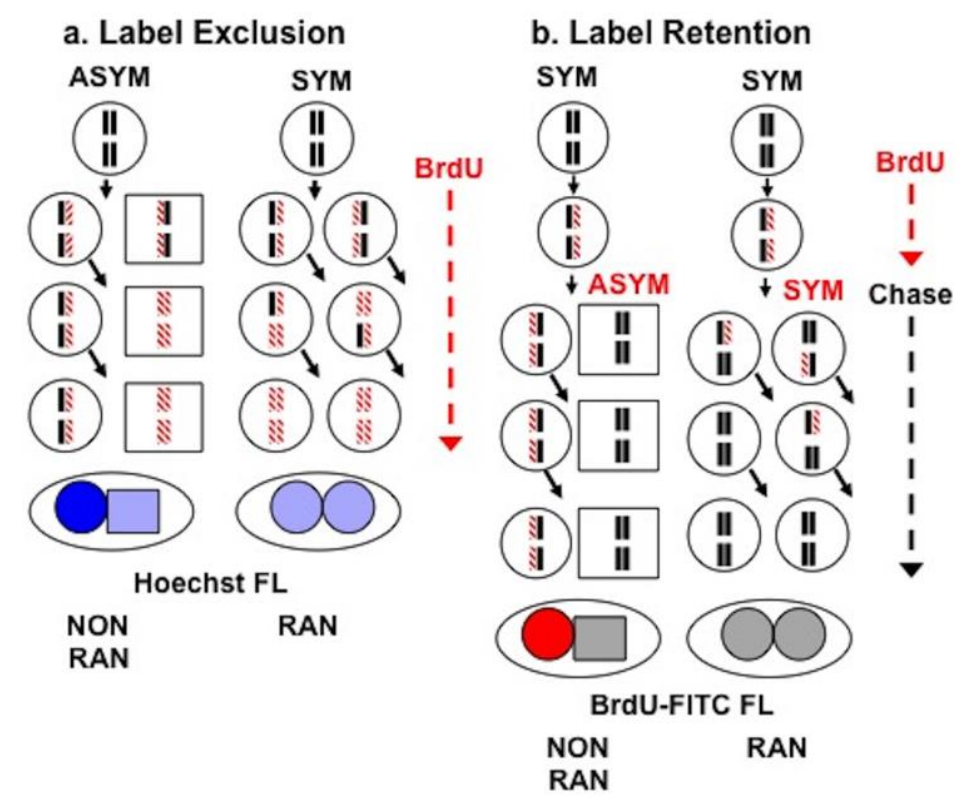

Figure 6. Schemes for detecting non-random sister chromatid segregation based on BrdU incorporation analyses. (a) Label exclusion introduces BrdU for uptake under conditions that promote asymmetric self-renewal divisions (ASYM) with immortal DNA strands already established. In this scenario, with continuous BrdU labeling, cells cycling symmetrically (SYM) with non-random sister chromatid segregation will achieve a uniformly high BrdU content in most DNA strands (red striped). After a subsequent cytochalasin D arrest, paired sister nuclei will show uniform and equivalent quenched Hoechst dye fluorescent (FL; light blue), indicative of random sister chromatid segregation (RAN). In contrast, cytochalasin D-arrested paired sister nuclei that result from non-random sister chromatid segregation (NON RAN) will be unequal for Hoescht fluorescence, because the DNA of the brighter nucleus (dark blue) will be only hemi-substituted with BrdU ("exclusion") due to its earlier continuous cosegregation of the set of sister chromatids with the older unlabeled template, immortal DNA strands. (b) Label retention introduces BrdU for uptake under conditions that promote symmetric self-renewal divisions (SYM) with random sister chromatid segregation. Cells are labeled for a full generation period to develop hemi-substituted chromosomes as a result of semiconservative DNA replication. At the end of this pulse labeling period, cells are either shifted to conditions that promote asymmetric self-renewal division (ASYM) or maintained under conditions for symmetric self-renewal divisions (SYM); and cultured thereafter under chase conditions that limit additional incorporation of BrdU. After sufficient cell generations to allow dilution and randomization of BrdU-substituted DNA strands as a result of random chromosome segregation in symmetrically cycling cells, cells are arrested with cytochalasin D to produce binucleated cells. FITC-conjugated fluorescent antiBrdU antibodies are used to determine the amount of BrdU present in the paired nuclei ("retention"). Random sister chromatid segregation yields paired nuclei with equivalent low levels of BrdU-FITC fluorescence (FL; gray) indicative of their low level of retained BrdU. In contrast, non-random sister chromatid cosegregation yields paired nuclei with unequal BrdU-FITC fluorescence, with one nucleus having a fluorescence level comparable to that of the initial pulse-labeled cells. The high fluorescence nucleus (red) corresponds to the sister cell retaining previously labeled, cosegregating immortal DNA strands. Ovals, cytochalasin D-arrested cells with paired nuclei. Circles, cycling cells or, in ovals, nuclei destined for cycling sister cells. Squares, non-cycling cells or, in ovals, nuclei destined for non-cycling sister cells. 
Figure 7 shows the results of quantitative fluorescence microscopy imaging of the Hoechst dye fluorescence intensity of the cell nuclei in cytochalsin D-arrested cells at the completion of a label exclusion analysis. Cytochalasin D allows nuclear division, but prevents cytokinesis. As such, it produces binucleated cells with two sister nuclei. The cytochalasin D arrest serves two important purposes for label exclusion analyses. First, by quantifying only the fluorescence intensity of nuclei in binucleated cells, the analysis was restricted to evaluation of only the cycling cells in a culture. This is an important restriction given the large number of non-dividing cells produced in 12(3) cultures (see Figures 4 and 5 and Table 2). Second, it provided a direct inspection of the segregation pattern of marked sister chromatids between sister cell nuclei.

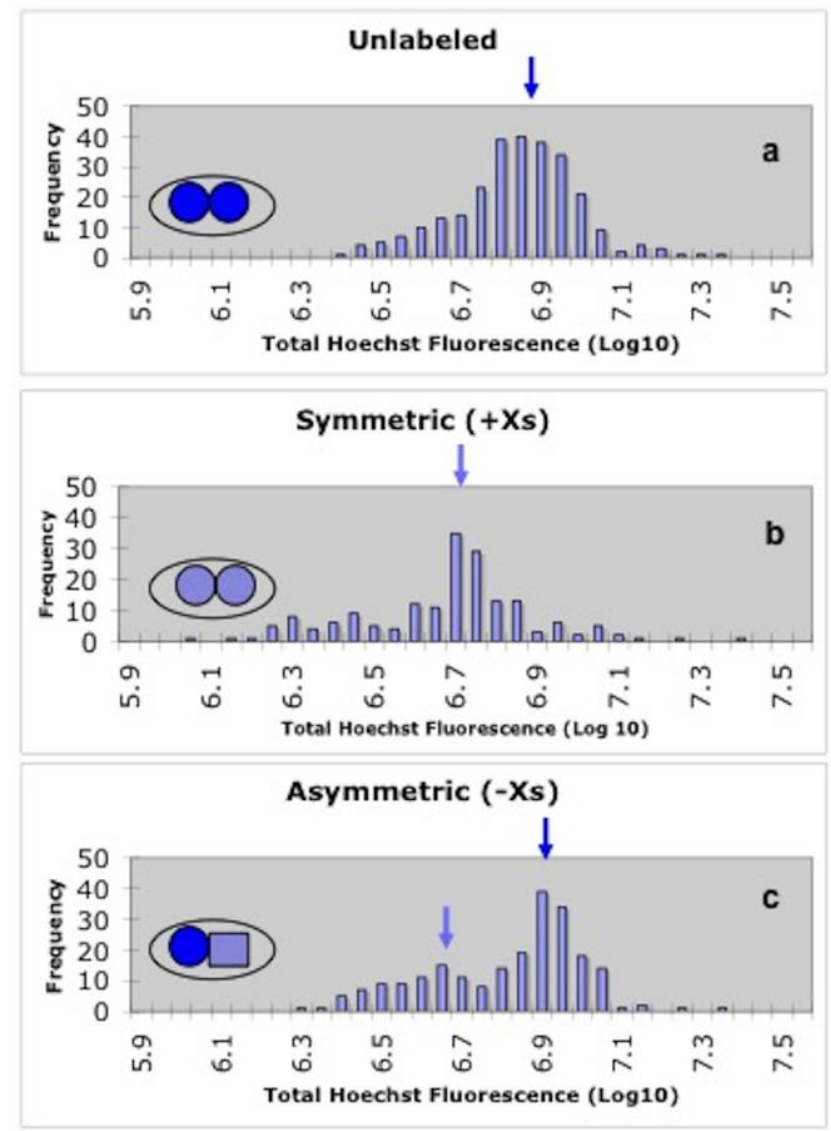

Figure 7. Label exclusion analysis of patterns of sister chromatid segregation in cultures of SACK agent-expanded human hepatic cell strain 12(3). Strain 12(3) cells were cultured continuously in BrdU for 2.5 cell generations as described in Figure 6a in either Xs-supplemented medium (b) (1.5 mM) - to promote symmetric self-renewal division—or Xs-free medium (c)—to promote asymmetric self-renewal divisions. In parallel, cells in Xs-supplemented medium were also cultured without BrdU for quantitative Hoescht fluorescence comparison. At the end of the continuous labeling period, all cells were treated with cytochalasin D to produce binucleated cells and then stained with Hoechst dye. Without regard to nuclear pairing, the individual Hoechst fluorescence of nuclei in binucleated cells in the three different types of cultures were quantified by fluorescence microscopy. Histograms report the frequencies of nuclei with the indicated fluorescence intensities. Inset drawings show the idealized expectations for fluorescence intensity patterns of nuclei in binucleates indicative of no BrdU label (a), uniform quenching as a result of random sister chromatid segregation (b), or unequal, asymmetric quenching resulting from non-random sister chromatid segregation (c). Ovals, cytochalasin D-arrested cells with paired nuclei. Circles, nuclei destined for cycling sister cells. Square, nucleus destined for a non-cycling sister cell. Dark blue, Hoechst bright. Light blue, Hoechst dim. Arrows indicate the median values of delineated unimodal $(\mathbf{a}, \mathbf{b})$ and bimodal (c) fluorescence intensity distributions. 
In the label exclusion design, the newly synthesized DNA of cycling cells is marked continuously by substitution of the thymidine analogue bromodeoxyuridine (BrdU) for several generations. Under these conditions, the DNA in cells randomly segregating sister chromatids approach complete bisubstitution with BrdU. This high level of BrdU substitution causes a quantifiable reduction in the fluorescence of Hoechst dye molecules intercalated in the DNA double helix caused by BrdU quenching. This effect is evident by comparing the distribution of the Hoechst fluorescence intensity of nuclei in binucleated cytochalsin D-arrested cells after as few as 2.5 cell generations of BrdU uptake. Figure 7a shows that the $\log$ fluorescence intensity distribution of strain 12(3) cells cultured in Xs-free medium without BrdU supplementation (median $=6.83$; mean $=6.82 \pm 0.16$ ). After continuous culture in Xs-supplemented medium with BrdU for 2.5 cell generations, the distribution of log fluorescence intensity shifts uniformly to a lower median fluorescence intensity (median $=6.68$; mean $=6.65 \pm 0.22$; Figure $7 \mathrm{~b}$ ), as predicted for random sister chromatid segregation.

In contrast, labeling with BrdU under Xs-free conditions produces a bimodal fluorescence intensity distribution (Figure 7c), with a peaks of log fluorescence intensities centered at the positions of both quenched (median = 6.65) and unquenched Hoechst fluorescence (median = 6.90). The distribution corresponding to quenched nuclei in binucleated cells cultured under Xs-free conditions appeared to be distinct from the distribution corresponding to the quenched nuclei of binucleated cells cultured in Xs-supplemented medium, having a 6.7\% lower median fluorescence intensity (Figure 7c). The shift to lower fluorescence intensity was predicted for nuclei in which all chromosomes had both strands substituted (i.e., bisubstituted) with BrdU. For random sister chromatid segregation, 2.5 generation of cycling in BrdU was not sufficient to produce such cells. For this labeling protocol, uniformly quenched cells were predicted to have mixtures of hemi-substituted chromosomes and bisubstituted chromosomes that averaged out to $75-87.5 \%$ total BrdU substitution. In marked contrast, non-random sister chromatid segregation was predicted to produce sister nuclei with chromosomes that were bisubstituted with BrdU after only two cell generations. The data in Figure 7c were consistent with this explanation.

The unquenched distribution of fluorescence intensity in Xs-free medium was predicted to be due to nuclei in binucleated cells that were undergoing non-random sister chromatid segregation. Due to maintaining a full genomic complement of hemi-substituted chromosomes with one unlabeled immortal DNA strand, the DNA in such nuclei would have less effective quenching of Hoechst fluorescence. To further evaluate this explanation for the data in Figure 7c, we quantified differences in the fluorescence intensity of paired nuclei in individual binucleated cells quantified in the label exclusion experiment. Non-random segregation would be indicated by significant number of paired nuclei that were unequal in fluorescence intensity. Unequal nuclear fluorescence intensities are predicted because bisubstituted chromatids, with both their complementary template and new DNA strands labeled with BrdU, cosegregate to the non-stem sister nucleus; while hemi-substituted chromatids, with an unlabeled immortal template strand and a BrdU-substituted new DNA strand, were predicted to cosegregate to the stem cell sister nucleus.

Figure 8 provides histograms of the \% difference in the log fluorescence intensity between paired nuclei in individual cytochalasin D-arrested binucleated cells. For cells maintained in Xs-free medium, which promotes asymmetric self-renewal divisions, $63 \%$ of nuclear pairs differed by $>3.6 \%$. The $3.6 \%$ value is an important landmark for comparison because it corresponds to the \% difference predicted by the median values of the quenched and unquenched distributions identified in Figure $7 \mathrm{c}(\%$ difference $=$ $\{[6.90-6.65] / 6.90\} \times 100 \%)[1,5,6]$. The binucleates of cells maintained in Xs-supplemented medium had fewer values that were $>3.6 \%(52 \%)$, consistent with conditions that reduced the number of asymmetrically cycling cells. However, this difference was not statistically significant. 


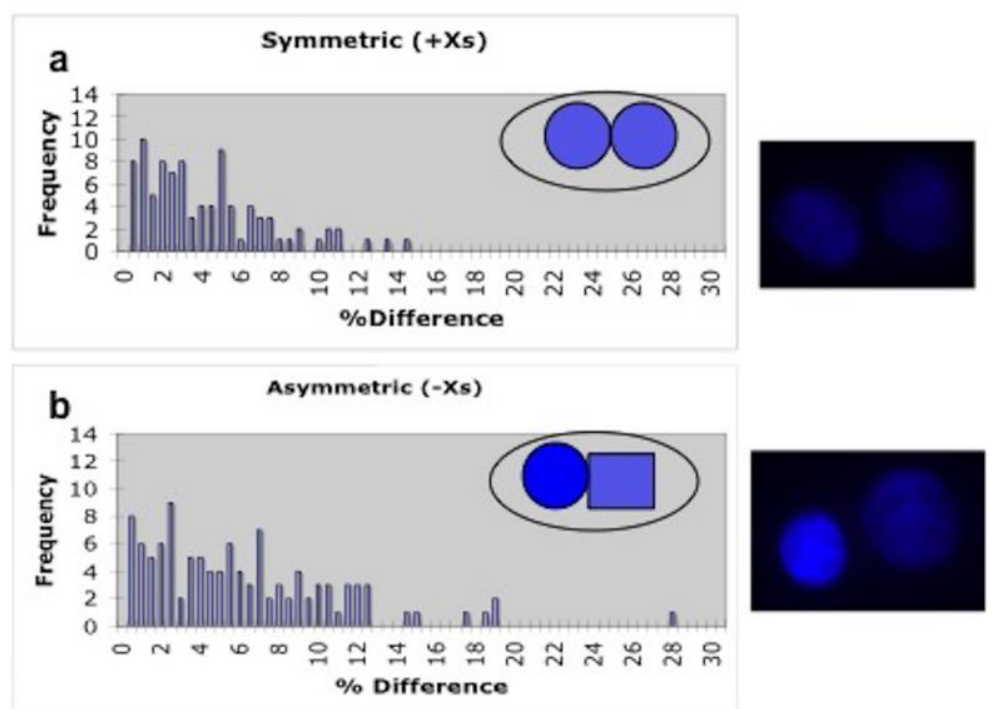

Figure 8. Quantitative analyses of the difference in the Hoechst dye fluorescence intensity of paired sister nuclei from cells from label exclusion analysis as described in Figure 6a. The Hoechst fluorescence intensities of cytochalasin D-arrested paired sister nuclei from the analysis in Figure 7 were determined by fluorescence microscopy. For each evaluated pair the $\%$ difference in fluorescence intensity was calculated $[1,5,6]$. The frequency distributions of $\%$ difference for cells labeled under Xs-supplemented conditions (a, $1.5 \mathrm{mM})$ - promoting symmetric self-renewal divisions-and Xs-free conditions (b) - promoting asymmetric self-renewal divisions-were compared. Inset drawings show the idealized expectations for fluorescence intensity patterns of nuclei in individual binucleated cells indicative of uniform quenching as a result of random sister chromatid segregation (a), or unequal, reduced quenching resulting from non-random sister chromatid segregation (b). Examples of fluorescence micrographs modeled by the idealized drawings are shown respectively. Ovals, cytochalasin D-arrested cells with paired nuclei. Circles, nuclei destined for cycling sister cells. Square, nucleus destined for a non-cycling sister cell. Dark blue, Hoechst bright. Light blue, Hoechst dim.

The label retention assay is a complementary approach to label exclusion for detection of non-random sister chromatid segregation. As diagrammed in Figure 6b, the label retention assay is initiated with a labeling period for one cell generation. In contrast to labeling in the label exclusion assay, BrdU labeling for label retention studies was performed under conditions that promote symmetric self-renewal and random sister chromatid segregation and for only one cell generation. Labeling was limited to one cell cycle to ideally produce cellular genomes for which chromosomes were hemi-substituted with BrdU. Thereafter, cells were shifted to conditions that promote asymmetric self-renewal kinetics and prevent further labeling with BrdU. After additional culture for several generations of asymmetric self-renewal divisions, cycling cells were arrested with cytochalsin $\mathrm{D}$ and fixed for immunofluorescence microscopy with fluorescent antiBrdU antibodies.

The distribution of antiBrdU fluorescence between paired sister nuclei is an indicator of the pattern of sister chromatid segregation in cells. Sister nuclei with equal lower levels of fluorescence compared to the starting level of fluorescence in cells immediately after the labeling period were predicted for random segregation (For examples, see Figure 9b). In marked contrast, finding sister nuclei that have unequal fluorescence-one with a level similar to initially labeled cells and the other with a level approaching background fluorescence-is indicative of non-random segregation (for examples, see Figure 9a).

In label exclusion experiments with strain 12(3) cells cultured during the post-labeling chase period under $\mathrm{Xs}_{\mathrm{s}-\mathrm{supp}}$ lemented conditions to promote symmetric self-renewal divisions, only $0.7 \%$ ( 2 of 281) of binucleated cells showed an unequal pattern of BrdU fluorescence. However, when cultured under $\mathrm{Xs}_{\mathrm{S}}$-free conditions to promote asymmetric self-renewal after the labeling period, 
$4.9 \%$ ( 18 of $368 ; p=0.0021)$ of binucleates showed unequal fluorescence of the type shown in Figure 9a. Although only 18 unequal binucleates were identified for the specific quantitative analyses, a total of 45 similarly unequal binucleated cells were observed overall.

a. UNEQUAL DAPI BrdU

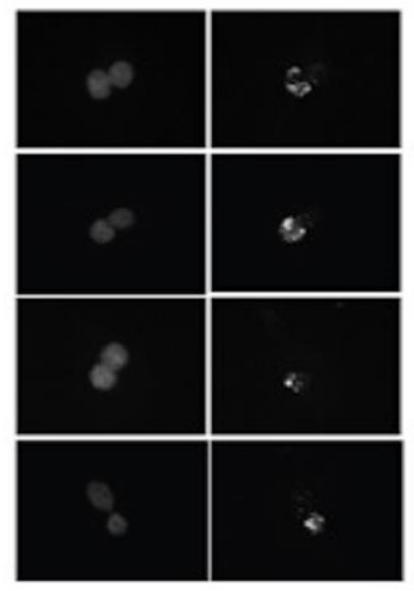

\section{b. EQUAL}
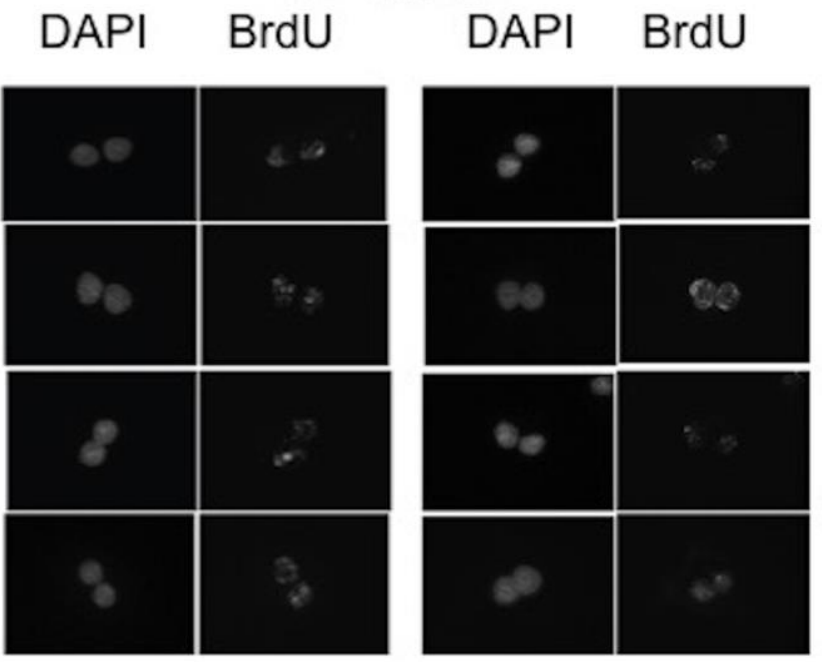

Figure 9. Label retention analysis of patterns of sister chromatid segregation in cultures of SACK agent-expanded human hepatic cell strain 12(3). Strain 12(3) cells, cultured in Xs-supplemented medium to promote symmetric self-renewal divisions, were pulse-labeled for $24 \mathrm{~h}$ with BrdU and analyzed for BrdU retention after $96 \mathrm{~h}$ of chase in either Xs-free medium (to promote asymmetric self-renewal divisions) or Xs-supplemented medium (to maintain symmetric self-renewal divisions), as described in Figure $6 \mathrm{~b}$. At the end of the chase period, cells were arrested as binucleates with cytochalasin D, fixed, and costained with FITC-conjugated fluorescent antiBrdU antibodies and DAPI to identify paired sister nuclei. (a) Examples of fluorescence micrographs of binucleated cells found in Xs-free cultures with only one nucleus showing a high BrdU content. (b) Examples of fluorescence micrographs of paired sister nuclei found in Xs-supplemented cultures showing equivalent lower BrdU content.

\section{Discussion}

The presented studies provide a more detailed characterization of the cell kinetics and chromosome segregation properties of SACK agent-derived human hepatic stem cell strains previously described in brief $[10,47]$. In particular, the properties of hepatic stem cell strain 12(3) and its clonal derivatives are described in greater detail. The SACK method does not achieve complete suppression of the asymmetric self-renewal of explanted tissue stem cells. However, the degree of suppression, which promotes exponential symmetric self-renewal of tissue stem cells, is sufficient in many cases to achieve the expansion and propagation of cell strains and clonal derivatives with high fractions of tissue stem cells, defined by SACK agent-dependent asymmetric self-renewal kinetics [5,6,43,45-47]. Recently, strain 12(3) hepatic tissue stem cells were studied to show that human carcinogens like benzene have SACK agent properties [10].

The 12(3) parental cell strain has several special characteristic that define it as originating from in vivo hepatic stem cells that have been expanded and propagated in culture. Evidence of the hepatic origin of the 12(3) strain begins with its establishment with cells isolated from normal donor human liver tissue. Under differentiation induction conditions (i.e., confluent cell density and reduced serum), 12(3) cells and clonally derived strains express and secrete the hepatocyte lineage-specific proteins albumin and alpha-fetoprotein [47]. Whereas albumin is expressed by both mature hepatocytes and hepatocyte precursors, high-level alpha-fetoprotein production is a hepatoblast phenotype. Indicative of the strain's tissue stem cell character, under conditions of active proliferation, these proteins are not secreted [47]. Mass spectrometry analyses of proteins secreted by the cells under differentiating 
conditions detected all the proteins that constitute the extracellular matrix of the human hepatocyte basement membrane (data not shown). Strain 12(3) cells also express the hepatocyte nuclear pregnane $X$ receptor (PXR) that regulates the transcription of cytochrome P450 3A4 [47].

The presented findings provided an independent verification that 12(3) cells were a tissue stem cell strain. Previous studies establish the SACK method as a procedure that yields propagating strains for tissue stem cells from rat liver [43], mouse hair follicle [5,6], and human pancreas [45,46]. A key attribute of SACK agent-derived tissue stem cell strains is asymmetric cell kinetics that are reversibly suppressible by the respective SACK agent used for derivation. SACK is characterized by asymmetrically self-renewing tissue stem cells shifting to exponential symmetric self-renewal. This change in cell kinetics accounts for the ability of SACK agents to extend the proliferative lifespan of primary human tissue cells [44].

The ability of Xs-supplementation to enable the clonal expansion of secondary strains of natural and genetically engineered 12(3) cells is another important demonstration of the cell strain's tissue stem cell character. The clonal strains retention of their Xs-dependent asymmetric cell kinetics indicates that they have not undergone genetic immortalization events, which are rare for human cells in any case. To the authors' knowledge, there are no previous reports of the establishment of clonal derivatives from primary human cell cultures without concomitant neoplastic transformation.

Several independent analyses were conducted to verify the SACK agent-dependent asymmetric cell kinetics of strain 12(3) cells. Like other compared SACK agent-derived cell strains, the colony formation efficiency of 12(3) cells was significantly increased by their respective SACK derivation agent, Xs. Colcemid arrest detected the production of non-cycling cells in 12(3) cultures, which are predicted for asymmetric self-renewal kinetics. Consistent with the SACK agent dependency of asymmetric stem cell kinetics, supplementation with Xs greatly reduced the production of non-cycling cells. Time-lapse microscopy confirmed that non-cycling cells were produced in asymmetric self-renewal division lineages. However, a significant effect of Xs was not detected on the frequency of asymmetric cell lineages or the frequency of individual asymmetric cell divisions observed by time-lapse. Failure to detect a significant difference commensurate with the colony formation and colcemid arrest analyses may be due to the inherently lower sample size of time-lapse microscopy studies.

Previously, we reported the use of two well-established complementary approaches to detect and quantify cells undergoing non-random sister chromatid segregation in engineered cells [1,4-8] and clonally derived, SACK agent-derived murine tissue stem cell strains [5-8]. The same strategies were applied to investigate sister chromatid DNA strand inheritance patterns in strain 12(3) cells, comparing conditions of Xs-supplemented culture and Xs-free culture. The label exclusion studies yield quantitative evidence of $X$ s-suppressible non-random sister chromatid segregation. Quantitative analyses of the quenching of Hoechst fluorescence in cytochalsin D-arrested binucleated cells after continuously labeling in BrdU indicated a significant fraction of cells with Xs-suppressible non-random segregation. The distribution of individually tabulated Hoechst fluorescence of nuclei in cycling cells identified by binucleation was dramatically different between Xs-supplemented and Xs-free cultures. In Xs-supplemented medium, promoting symmetric renewal kinetics, the fluorescence intensity distribution was consistent with uniform quenching, indicative of random sister chromatid segregation (compare Figure 7b to Figure 7a). However, for Xs-free medium that promotes asymmetric cell kinetics, the distribution was bimodal, with significant distributions at both the position of quenched and unquenched Hoechst fluorescence (Figure $7 \mathrm{c}$ ). This pattern was consistent with a significant fraction of cells undergoing non-random segregation.

Close scrutiny of the label exclusion histogram data (Figure 7) might suggest a quantitative inconsistency. The median fluorescence intensity of the unquenched distribution identified after BrdU labeling (Figure $7 \mathrm{c}$; median $=6.9$ ) was quantitatively greater than the median of the distribution of unlabeled cells (Figure 7a; median $=6.83$ ). After BrdU incorporation for 2.5 cell generations, theoretically, there should be no cycling cells that are BrdU-free, indicated by the maximum level of Hoechst fluorescence under BrdU-free conditions (Figure 7a). Theorized stem cell nuclei cosegregating 
a complement of unlabeled immortal DNA strands were predicted to still show a degree of quenching due to the hemi-substitution of their retained chromosomes with BrdU. However, because of the small amount of the predicted degree of quenching (1.3\%) the analysis performed lacked the statistical resolution needed to evaluate this expectation. Subsequent comparison of the fluorescence of sister nuclei in individual cytocholasin D-arrested binucleated cells showed a high fraction with a degree of difference expected for non-random segregation. Though more cases of unequal fluorescence were observed for cells in Xs-free medium, the difference was not statistically significant.

The second DNA strand inheritance analysis, label-retention, provides qualitatively and quantitatively superior evidence of non-random sister chromatid segregation. In the presented studies, it also provided a more robust demonstration of the Xs-dependence of non-random segregation by strain 12(3) human hepatic stem cells. In label retention analyses, the hallmark of non-random sister chromatid segregation is continuous asymmetric, unequal inheritance of previously labeled BrdU-substituted DNA strands. Under Xs-free conditions, 4.9\% of 12(3) cells exhibited a BrdU detection pattern indicative of non-random segregation (Figure 9a, unequal). Xs-supplementation reduced the fraction of cells with the unequal BrdU detection pattern by $86 \%(0.7 \%)$. Based on the high amount of BrdU-labeled DNA retained after one cell generation of labeling, the data also indicate that newly synthesized DNA strands become immortal stands when asymmetric self-renewal was initiated. This intriguing mechanistic feature of the non-random segregation process has been consistently observed in previous demonstrations of non-random sister chromatid segregation in murine cells [1-8].

Cairns proposed the immortal DNA strand hypothesis to resolve a mathematical inconsistency between projected rates of spontaneous DNA replication errors in human tissue cells and observed rates of human cancers [11-13]. He noted that cancer rates were lower than expected. To account for the seeming discrepancy, he proposed that the active turnover of most mature tissue cells made them infrequent cells of origin for cancers. Instead, because of their longer lifetimes in human tissues, he attributed the origin of the majority of cancers to mutated tissue stem cells. He went on to invoke a unique mechanism, specifically in tissue stem cells in their basal state of asymmetric self-renewal of mature tissue cells, which would greatly reduce their accumulation of carcinogenic mutations. Cairns called this unique mechanism non-random sister chromatid segregation; and he coined the term immortal DNA strands for DNA strands unique to tissue stem cells that this mechanism kept free of mutations.

Cairns based his proposal on the work of Lark [39-42], who had reported earlier the first evidence of non-random sister chromosome segregation in both mammalian cells and plants. Prescient in Lark's studies, non-random segregation was detected in primary mammalian cell cultures, but not in cultures of tumor-derived cells [41]. It is now clear that this seminal distinction, which supported Cairns' idea that immortal strand cosegregation prevented the development of cancers, reflects the tight association between non-random sister chromatid segregation and the asymmetric self-renewal of tissue stem cells. Generally, tumor-derived cell lines are deficient for asymmetric self-renewal division [15].

Our earlier studies established an important synthesis of the discovery of Lark, the genetic fidelity ideas of Cairns, and more recent advances in understanding cancer mechanisms. In addition to confirming Cairns' vision that non-random sister chromatid segregation would only occur with asymmetric self-renewal division, we showed that both processes require normal expression of the TP53 protein. Mutations that cause loss of normal TP53 function are now well established as the most common genetic mutation in many diverse types of human cancer, and in particular those that arise in tissues with high rates of asymmetric self-renewal by tissue stem cells [14-16].

Despite this informative connection to p53 gene mutation, there is still a general state of disregard for Lark's seminal discovery of non-random sister chromatid segregation and Cairns' subsequent proposal that non-random segregation is an essential cellular mechanism for limiting the emergence of cancers before reproductive maturity in humans, and possibly other mammalians and vertebrates as well. This state continues despite many reports of evidence for non-random sister chromatid segregation in murine tissues [9,19-23] and murine tissue cells [1-9,22,23] including well-defined 
murine tissue stem cells [5-9,22,23]. In the present report, we extend this evidence to normal human tissue stem cells. This advance provides a next valuable step to support Cairns' prediction that such a mechanism would be found in human tissues; and prepares the way for direct investigations of the tissue stem cell genetic fidelity prediction of the immortal strand hypothesis.

\section{Conclusions}

Experimental evidence is presented for non-random sister chromatid segregation in normal human tissue stem cells in culture. The evaluated cells were a strain of human hepatic stem cells, designated as 12(3). Strain 12(3) cells were derived by the method of suppression of asymmetric cell kinetics (SACK), using the SACK agent xanthosine (Xs). An intrinsic feature of the SACK method is the expansion and propagation of tissue stem cells that exhibit SACK agent-dependent asymmetric self-renewal kinetics. In his seminal proposal, Cairns postulated that non-random cosegregation of sister chromatids, bearing the oldest immortal template DNA strands in tissue stem cells, would only occur when stem cells underwent asymmetric self-renewal divisions. We reported evidence that 12(3) cells possess the property of Xs-suppressible asymmetric self-renewal kinetics. Using BrdU-labeled DNA strand inheritance analyses, we presented complementary evidence of non-random sister chromatid segregation by 12(3) human hepatic stem cells associated with conditions that promoted their asymmetric self-renewal. This advance is an important step in the evaluation of Cairns' proposal that immortal DNA strand cosegregation is a key mechanism that limits the accumulation of spontaneous carcinogenic mutations in human tissue stem cells.

\section{Patents}

Sherley, J.L.; Panchalingam, K. Methods for ex vivo propagation of adult hepatic stem cells. 2010, U.S. Patent No. 7,824,912 B2.

Author Contributions: Authors contributions were conceptualization, J.L.S. and K.P.; methodology, K.P., L.J., B.D.C.; validation, K.P., L.J., B.D.C., and J.L.S.; formal analysis, J.L.S., K.P., L.J., and B.D.C.; investigation, J.L.S., K.P., L.J., and B.C; data curation, J.L.S., K.P., L.J., and B.D.C.; writing-original draft preparation, J.L.S., K.P., L.J., and B.D.C; writing-review and editing, J.L.S., K.P., L.J., and B.D.C.; visualization, J.L.S., K.P., L.J., and B.D.C.; supervision, J.L.S.; project administration, J.L.S.; funding acquisition, J.L.S., and K.P. All authors have read and agreed to the published version of the manuscript.

Funding: This research was funded by National Institutes of Health-National Institute of General Medical Sciences, Director's Pioneer Award 5DP1OD000805.

Acknowledgments: We thank Janice Lansita, Jean-François Paré, Rouzbeh Taghizadeh, and Gracy Crane for technical consultation and helpful critical discussions during the course of these studies.

Conflicts of Interest: The authors declare no conflict of interest. The funders had no role in the design of the study; in the collection, analyses, or interpretation of data; in the writing of the manuscript, or in the decision to publish the results.

\section{References}

1. Merok, J.R.; Lansita, J.A.; Tunstead, J.R.; Sherley, J.L. Co-segregation of chromosomes containing immortal DNA strands in cells that cycle with asymmetric stem cell kinetics. Cancer Res. 2002, 62, 6791-6795. [PubMed]

2. Lansita, J.A.; Merok, J.R.; Sherley, J.L. Physicochemical demonstration of immortal DNA strand segregation. In Mechanisms of Toxicity, Carcinogenesis, Cancer Prevention and Cancer Therapy Abstracts of the Seventeenth Aspen Cancer Conference. Toxicol. Pathol. 2003, 31, 163-164.

3. Merok, J.R.; Tunstead, J.R.; Lansita, J.A.; Sherley, J.L. Demonstration of an immortal DNA strand mechanism in cells that cycle with asymmetric stem cell kinetics-implications for mechanisms of human cancer and aging. In Mechanisms of Toxicity, Carcinogenesis, Cancer Prevention and Cancer Therapy: Abstracts of the Seventeenth Aspen Cancer Conference. Toxicol. Pathol. 2003, 31, 165-166.

4. Rambhatla, L.; Ram-Mohan, S.; Cheng, J.J.; Sherley, J.L. Immortal DNA strand co-segregation requires p53/IMPDH-dependent asymmetric self-renewal associated with adult stem cells. Cancer Res. 2005, 65, 3155-3161. [CrossRef] [PubMed] 
5. Huh, Y.H.; Sherley, J.L. Molecular Cloaking of H2A.Z on Mortal DNA Chromosomes During Nonrandom Segregation. STEM Cells 2011, 29, 1620-1627. [CrossRef] [PubMed]

6. Huh, Y.H.; King, J.; Cohen, J.; Sherley, J.L. SACK-Expanded Hair Follicle Stem Cells Display Asymmetric Nuclear Lgr5 Expression with Non-Random Sister Chromatid Segregation. Sci. Rep. 2011, 1, 176. [CrossRef] [PubMed]

7. Huh, Y.H.; Cohen, J.; Sherley, J.P.J. Higher 5-hydroxymethylcytosine identifies immortal DNA strand chromosomes in asymmetrically self-renewing distributed stem cells. Proc. Natl. Acad. Sci. USA 2013, 110, 16862-16867. [CrossRef]

8. Huh, Y.H.; Sherley, J.P.J. Decreased H3K27 and H3K4 trimethylation on mortal chromosomes in distributed stem cells. Cell Death Dis. 2014, 5, e1554. [CrossRef]

9. Huh, Y.H.; Noh, M.; Burden, F.; Chen, J.C.; Winkler, D.A.; Sherley, J.L. Use of sparse feature bioinformatics to identify a novel pattern-specific biomarker for counting asymmetrically self-renewing distributed stem cells. Stem Cell Res. 2015, 14, 144-154. [CrossRef]

10. Panchalingam, K.; Noh, M.; Huh, Y.H.; Sherley, J.L. Distributed Stem Cell Kinetotoxicity: A New Concept to Account for the Human Carcinogenicity of Non-Genotoxic Toxicants. In Human Stem Cell Toxicology, Issues in Toxicology No. 29; Sherley, J.L., Ed.; Royal Society of Chemistry: London, UK, 2016; pp. 250-279.

11. Cairns, J. Mutation selection and the natural history of cancer. Nat. Cell Biol. 1975, 255, 197-200. [CrossRef]

12. Cairns, J. Somatic stem cells and the kinetics of mutagenesis and carcinogenesis. Proc. Natl. Acad. Sci. USA 2002, 99, 10567-10570. [CrossRef] [PubMed]

13. Cairns, J. Cancer and the Immortal Strand Hypothesis. Genet 2006, 174, 1069-1072. [CrossRef] [PubMed]

14. Sherley, J. Mechanisms of Genetic Fidelity in Mammalian Adult Stem Cells. In Tissue Stem Cells; Potten, C.S., Clarke, R.B., Wilson, J., Renehan, A.G., Eds.; Taylor Francis: New York, NY, USA, 2006; pp. 37-54.

15. Sherley, J.L. New cancer diagnostics and therapeutics from a 9th "hallmark of cancer": Symmetric self-renewal by mutated distributed stem cells. Expert Rev. Mol. Diagn. 2013, 13, 797-810. [CrossRef] [PubMed]

16. Sherley, J.L. The p53 tumor suppressor gene as regulator of somatic stem cell renewal division. Cope 1996, 12, 9-10.

17. Karpowicz, P.; Morshead, C.M.; Kam, A.; Jervis, E.; Ramunas, J.; Cheng, V.; Van Der Kooy, D. Support for the immortal strand hypothesis: Neural stem cells partition DNA asymmetrically in vitro. J. Cell Biol. 2005, 170, 721-732. [CrossRef]

18. Sundararaman, B.; Avitabile, D.; Konstandin, M.H.; Cottage, C.T.; Gude, N.; Sussman, M.A. Asymmetric chromatid segregation in cardiac progenitor cells is enhanced by Pim-1 kinase. Circ. Res. 2012, 110, 1169-1173. [CrossRef]

19. Potten, C.S.; Hume, W.J.; Reid, P.; Cairns, J. The segregation of DNA in epithelial stem cells. Cell 1978, 15, 899-906. [CrossRef]

20. Potten, C.S.; Owen, G.; Booth, D. Intestinal stem cells protect their genome by selective segregation of template DNA strands. J. Cell Sci. 2002, 115, 2381-2388.

21. Smith, G.H. Label-retaining epithelial cells in mouse mammary gland divide asymmetrically and retain their template DNA strands. Development 2005, 132, 681-687. [CrossRef]

22. Shinin, V.; Gayraud-Morel, B.; Gomès, D.; Tajbakhsh, S. Asymmetric division and cosegregation of template DNA strands in adult muscle satellite cells. Nat. Cell Biol. 2006, 8, 677-682. [CrossRef]

23. Conboy, M.J.; Karasov, A.O.; Rando, T.A. High incidence of non-random template strand segregation and asymmetric fate determination in dividing stem cells and their progeny. PLoS ONE 2007, 5, e102.

24. Kajstura, J.; Bai, Y.; Cappetta, N.; Kim, J.; Arranto, C.; Sanada, F.; D’Amario, M.; Matsuda, A.; Bardelli, S.; Ferreira-Martins, J.; et al. Tracking chromatid segregation to identify human cardiac stem cells that regenerate extensively the infarcted myocardium. Circ. Res. 2012, 111, 894-906. [CrossRef] [PubMed]

25. Pine, S.R.; Ryan, B.M.; Varticovski, L.; Robles, A.I.; Harris, C.C. Microenvironmental modulation of asymmetric cell division in human lung cancer cells. Proc. Natl. Acad. Sci. USA 2010, 107, 2195-2200. [CrossRef] [PubMed]

26. Bussard, K.M.; Boulanger, C.A.; Kittrell, F.S.; Behbod, F.; Medina, D.; Smith, G.H. Immortalized, premalignant epithelial cell populations contain long-lived, label-retaining cells that asymmetrically divide and retain their template DNA. Breast Cancer Res. 2010, 12, R86. [CrossRef] [PubMed] 
27. Hari, D.; Xin, H.-W.; Jaiswal, K.; Wiegand, G.; Kim, B.-K.; Ambe, C.; Burka, D.; Koizumi, T.; Ray, S.; Garfield, S.; et al. Isolation of Live Label-Retaining Cells and Cells Undergoing Asymmetric Cell Division via Nonrandom Chromosomal Cosegregation from Human Cancers. Stem Cells Dev. 2011, 20, 1649-1658. [CrossRef] [PubMed]

28. Xin, H.-W.; Hari, D.M.; Mullinax, J.E.; Ambe, C.M.; Koizumi, T.; Ray, S.; Anderson, A.J.; Wiegand, G.W.; Garfield, S.H.; Thorgeirsson, S.S.; et al. Tumor-Initiating Label-Retaining Cancer Cells in Human Gastrointestinal Cancers Undergo Asymmetric Cell Division. Stem Cells 2012, 30, 591-598. [CrossRef] [PubMed]

29. Liu, W.; Jeganathan, G.; Amiri, S.; Morgan, K.M.; Ryan, B.M.; Pine, S.R. Asymmetric segregation of template DNA strands in basal-like human breast cancer cell lines. Mol. Cancer 2013, 12, 139. [CrossRef]

30. Rosenberger, R.F.; Kessel, M. Nonrandom Sister Chromatid Segregation and Nuclear Migration in Hyphae of Aspergillus nidulans. J. Bacteriol. 1968, 96, 1208-1213. [CrossRef]

31. Karpowicz, P.; Pellikka, M.; Chea, E.; Godt, D.; Tepass, U.; Van Der Kooy, D. The germline stem cells of Drosophila melanogaster partition DNA non-randomly. Eur. J. Cell Biol. 2009, 88, 397-408. [CrossRef]

32. Yadlapalli, S.; Yamashita, Y.M. Chromosome-specific nonrandom sister chromatid segregation during stem-cell division. Nat. Cell Biol. 2013, 498, 251-254. [CrossRef]

33. Kiel, M.J.; He, S.; Ashkenazi, R.; Gentry, S.N.; Teta, M.; Kushner, J.A.; Jackson, T.L.; Morrison, S.J. Haematopoietic stem cells do not asymmetrically segregate chromosomes or retain BrdU. Nat. Cell Biol. 2007, 449, 238-242. [CrossRef] [PubMed]

34. Sotiropoulou, P.A.; Candi, A.; Blanpain, C. The Majority of Multipotent Epidermal Stem Cells Do Not Protect Their Genome by Asymmetrical Chromosome Segregation. Stem Cells 2008, 26, 2964-2973. [CrossRef] [PubMed]

35. Waghmare, S.K.; Bansal, R.; Lee, J.; Zhang, Y.V.; McDermitt, D.J.; Tumbar, T. Quantitative proliferation dynamics and random chromosome segregation of hair follicle stem cells. EMBO J. 2008, 27, 1309-1320. [CrossRef] [PubMed]

36. Escobar, M.; Nicolas, P.; Sangar, F.; Laurent-Chabalier, S.; Clair, P.; Joubert, D.; Jay, P.; Legraverend, C. Intestinal epithelial stem cells do not protect their genome by asymmetric chromosome segregation. Nat. Commun. 2011, 2, 258. [CrossRef] [PubMed]

37. Yadlapalli, S.; Cheng, J.; Yamashita, Y.M. Drosophila male germline stem cells do not asymmetrically segregate chromosome strands. J. Cell Sci. 2011, 124, 933-939. [CrossRef]

38. Steinhauser, M.L.; Bailey, A.P.; Senyo, S.E.; Guillermier, C.; Perlstein, T.S.; Gould, A.P.; Lee, R.T.; Lechene, C.P. Multi-isotope imaging mass spectrometry quantifies stem cell division and metabolism. Nat. Cell Biol. 2012, 481, 516-519. [CrossRef]

39. Lark, K.G. Nonrandom Segregation of Sister Chromatids. In Genetics E Developmental Biology; Teas, H.J., Ed.; University of Kentucky Press: Lexington, KY, USA, 1969; pp. 8-24.

40. Lark, K.G. Discovering non-random segregation of sister chromatids: The naïve treatment of a premature discovery. Front. Oncol. 2013, 2, 211. [CrossRef]

41. Lark, K.G.; Consigli, R.A.; Minocha, H.C. Segregation of Sister Chromatids in Mammalian Cells. Science 1966, 154, 1202-1205. [CrossRef]

42. Lark, K.G. Nonrandom segregation of sister chromatids in Vicia faba and Triticum boeoticum. Proc. Natl. Acad. Sci. USA 1967, 58, 352-359. [CrossRef]

43. Lee, H.-S.; Crane, G.G.; Merok, J.R.; Tunstead, J.R.; Hatch, N.L.; Panchalingam, K.; Powers, M.J.; Griffith, L.G.; Sherley, J.L. Clonal expansion of adult rat hepatic stem cell lines by suppression of asymmetric cell kinetics (SACK). Biotechnol. Bioeng. 2003, 83, 760-771. [CrossRef]

44. Paré, J.; Sherley, J.L. Biological Principles for Ex Vivo Adult Stem Cell Expansion. In Current Topics in Developmental Biology; Schatten, G., Ed.; Elsevier, Inc.: San Diego, CA, USA, 2006; Volume 73, pp. 141-171.

45. Paré, J.-F.; Sherley, J.L. Culture Environment-Induced Pluripotency of SACK-Expanded Tissue Stem Cells. J. Biomed. Biotechnol. 2011, 2011, 312457. [CrossRef] [PubMed]

46. Sherley, J.P.J. Ex vivo Expansion of Human Adult Pancreatic Cells with Properties of Distributed Stem Cells by Suppression of Asymmetric Cell Kinetics. J. Stem Cell Res. Ther. 2013, 3, 149. [CrossRef] [PubMed]

47. Sherley, J.L.; Panchalingam, K. Methods for Ex Vivo Propagation of Adult Hepatic Stem Cells. U.S. Patent No. 7,824,912 B2, 2 November 2010. 
48. Rambhatla, L.; Bohn, S.A.; Stadler, P.B.; Boyd, J.T.; Coss, R.A.; Sherley, J.L. Cellular senescence: Ex vivo p53-dependent asymmetric cell kinetics. J. Biomed. Biotechnol. 2001, 1, 28-37. [CrossRef] [PubMed]

49. Taghizadeh, R.R.; Sherley, J.L. CFP and YFP, but Not GFP, Provide Stable Fluorescent Marking of Rat Hepatic Adult Stem Cells. J. Biomed. Biotechnol. 2008, 2008, 453590. [CrossRef]

50. Sherley, J.L. Asymmetric self-renewal: The mark of the adult stem cell. In Stem Cell Repair and Regeneration; Habib, N.A., Gordon, M.Y., Levicar, N., Jiao, L., Thomas-Black, G., Eds.; Imperial College Press: London, UK, 2005; pp. 21-28.

51. Dutton, R.; Abdi, F.; Minnetyan, L.; Sherley, J.L.; Asymmetrex, L. A Computational Simulation Technology for Specific Counting of Perinatal and Postnatal Human Tissue Stem Cells for Transplantation Medicine. OBM Transplant. 2020, 4, 1-24. [CrossRef]

Publisher's Note: MDPI stays neutral with regard to jurisdictional claims in published maps and institutional affiliations.

(C) 2020 by the authors. Licensee MDPI, Basel, Switzerland. This article is an open access article distributed under the terms and conditions of the Creative Commons Attribution (CC BY) license (http://creativecommons.org/licenses/by/4.0/). 